\title{
Каскадный захват электронов на заряженные диполи в слабо компенсированных полупроводниках
}

\author{
(C) В.Я. Алешкин, Л.В. Гавриленко \\ Институт ффизики микроструктур Российской академии наук, \\ 603087 Нижний Новгород, Россия \\ E-mail: aleshkin@ipmras.ru
}

(Получена 27 апреля 2017 г. Принята к печати 12 мая 2017 г.)

Вычислены времена каскадного захвата электрона на заряженный диполь донор-акцептор для случая импульсного и стационарного возбуждений примесной фотопроводимости в $\mathrm{GaAs}, \mathrm{Ge}$ и $\mathrm{Si}$. Показано, что при концентрации заряженной примеси, большей $10^{13} \mathrm{~cm}^{-3}$, зависимость частоты каскадного захвата от концентрации заряженной примеси становится сублинейной в рассматриваемых полупроводниках.

DOI: $10.21883 /$ FTP.2017.11.45098.12

\section{1. Введение}

Хорошо известно [1], что в слабо компенсированных полупроводниках при достаточно низких температурах большая часть заряженной примеси располагается в комплексах, представляющих собой близко расположенные заряженные донор и акцептор. В этом случае каскадный захват электронов на заряженные примеси происходит в поле диполя [2]. Теория каскадного захвата электронов на заряженные диполи для случая стационарного возбуждения была разработана в работе [2] (см. [3]).

В недавней работе [4] было показано, что в случае импульсного возбуждения фотоэлектронов время захвата электрона на заряженный центр в кулоновском поле может значительно (более чем на порядок) отличаться от времени захвата, рассчитанного для стационарного случая. Такое большое различие времен захвата имеет место при достаточно больших концентрациях заряженной примеси, когда функция распределения фотоэлектронов в зоне проводимости может заметно отличаться от равновесной (максвелловской). Возникает естественный вопрос - насколько велико различие во временах каскадного захвата для случаев стационарного и импульсного возбуждения в том случае, когда захват идет в поле диполя. Цель настоящей работы - найти ответ на этот вопрос. Для этого были рассчитаны времена каскадного захвата электронов на заряженные доноры в слабо компенсированных $\mathrm{GaAs}$, Ge и $\mathrm{Si}$ при низких температурах, когда захват осуществляется в поле диполя в условиях импульсного и стационарного возбуждения.

\section{2. Модель для вычисления времени каскадного захвата}

Для нахождения времен каскадного захвата используем численное решение уравнения Больцмана. Как известно, каскадный захват происходит благодаря испусканию электроном акустических фононов. Имеется две трудности в вычислении времени каскадного захвата.
Первая трудность состоит в том, чтобы корректно учесть влияние потенциала заряженных примесей на вероятность рассеяния на фононах электронов с энергиями, близкими к порогу протекания. Вторая трудность состоит в вычислении плотности состояний в области делокализованных и локализованных состояний. Способ преодоления первой трудности был предложен Абакумовым, Перелем и Яссиевич [2]. Состоит он в применении подхода, предложенного Питаевским при рассмотрении электронной рекомбинации в одноатомном газе [5]. Суть этого метода заключается в усреднении вероятности с микроканоническим распределением по состояниям электрона после рассеяния. В этом случае для электрона, двигающегося в потенциале $U(r)$, вероятность рассеяния из состояния с энергией $E$ в состояние $E^{\prime}$ $\left(E>E^{\prime}\right)$ имеет вид [3]

$$
W\left(E, E^{\prime}\right)=\frac{\int \delta\left(E^{\prime}-\varepsilon^{\prime}-U(r)\right) w\left(\varepsilon \varepsilon^{\prime}\right) \sqrt{\varepsilon^{\prime}} d \varepsilon^{\prime} d^{3} r}{\int \delta\left(E^{\prime}-\varepsilon^{\prime}-U(r)\right) \sqrt{\varepsilon^{\prime}} d \varepsilon^{\prime} d^{3} r},
$$

где $w\left(\varepsilon, \varepsilon^{\prime}\right)$ - вероятность рассеяния свободного электрона из состояния с кинетической энергией $\varepsilon$ в состояние с кинетической энергией $\varepsilon^{\prime}$ (выражения для случая рассеяния на деформационном и пьезоакустическом потенциале акустических фононов можно найти в [6]). Интегрирование по координате в (1) ведется по доступной для электрона с энергией $E^{\prime}$ области. Отметим, что поскольку имеется много состояний с энергией $E^{\prime}$, то под $W\left(E, E^{\prime}\right)$ понимается усредненная по всем конечным состояниям вероятность. В [4] было показано, что $W\left(E, E^{\prime}\right)$ удовлетворяет принципу детального равновесия, если ему удовлетворяет $w\left(\varepsilon, \varepsilon^{\prime}\right)$ :

$$
W\left(E, E^{\prime}\right)=\exp \left(\frac{E-E^{\prime}}{k_{\mathrm{B}} T}\right) W\left(E^{\prime}, E\right),
$$

где $k_{\mathrm{B}}-$ постоянная Больцмана, $T-$ температура. Такой подход учета влияния потенциальной энергии электрона на вероятность рассеяния справедлив в случае, когда функция распределения электронов зависит только от энергии и не зависит от координат и импульса электрона. 
Чтобы преодолеть вторую трудность, упростим задачу аналогично тому, как это было сделано в работе [4]. Для этого будем полагать, что пары заряженные донор-акцептор расположены в кристалле не случайно, а регулярным образом - в центре кубов с длиной ребра $2 R_{0}=\left(N_{D}^{+}\right)^{-1 / 3}$, где $N_{D}^{+}-$концентрация заряженных доноров $\left(N_{D}-\right.$ концентрация доноров). Будем полагать, что заряженный акцептор находится от донора на расстоянии $d=N_{D}^{-1 / 3}(3 / 4 \pi)^{1 / 3} \Gamma(4 / 3) \approx 0.554 N_{D}^{-1 / 3}$, которое соответствует среднему расстоянию между ближайшими заряженными донорами и акцепторами [1]. Для упрощения вычисления вероятностей (1) сделаем еще одно допущение, полагая, что внутри куба электроны не могут выходить за пределы сферы с радиусом $R_{0}$. Как известно [3], основную роль играют в захвате электроны, энергия которых отличается от энергии уровня протекания (энергии, разделяющей локализованные и делокализованные состояния) на величину не более $k_{\mathrm{B}} T$. Будем полагать, что такие электроны двигаются в поле диполя, т. е. $U(r, \vartheta)=e^{2} d \cos \vartheta / \kappa r^{2}$, где $\kappa-$ статическая диэлектрическая проницаемость полупроводника, $e-$ заряд электрона, $\vartheta-$ угол между линией, соединяющей пару заряженный донор-акцептор, и радиусом-вектором электрона. Отметим, что это приближение работает при выполнении условия $e^{2} / \kappa d \gg k_{\mathrm{B}} T$.

Плотность состояний без учета спина электрона в одном кубе такой модели имеет вид

$$
g(E)=\frac{(2 m)^{3 / 2} R_{0}^{3} E_{0}^{3 / 2}}{15 \pi \hbar^{3}|E|} f_{1}(E),
$$

где $m$ - масса свободного электрона, $E_{0}=e^{2} d / \kappa R_{0}^{2}$, а функция $f_{1}(E)$ имеет вид

$$
f_{1}(E)=\left\{\begin{array}{lc}
1, & E<-E_{0} \\
1-\left(1-\frac{|E|}{E_{0}}\right)^{5 / 2}, & -E_{0}<E<0 \\
\left(\frac{E}{E_{0}}+1\right)^{5 / 2}-1, & 0<E<E_{0} \\
\left(\frac{E}{E_{0}}+1\right)^{5 / 2}-\left(\frac{E}{E_{0}}-1\right)^{5 / 2}-1, & E>E_{0} .
\end{array}\right.
$$

Из (4) видно, что плотность состояний непрерывна.

Для вычисления вероятностей рассеяния (1) полезно выразить плотность состояний через объем $v$, в котором может двигаться электрон:

$$
g(E)=\frac{(2 m)^{3 / 2} \sqrt{|E|}}{4 \pi^{2} \hbar^{3}} v f_{2}(E) .
$$

Выражение для безразмерной функции $f_{2}(E)$ приведено в Приложсении. Поскольку при $E \rightarrow \infty, f_{2}(E) \rightarrow 2$, то (5) переходит в плотность состояний для свободной частицы.

В рассматриваемой модели электроны могут перемещаться между кубами, если их энергия больше 0, т.е. это энергия, разделяющая делокализованные и локализованные состояния.
Для численного решения уравнения Больцмана интервал энергий $(-6,40)$ мэВ был разбит на 1150 одинаковых ячеек. Решение уравнения Больцмана сводится к решению системы уравнений, описывающих баланс электронов в ячейках [4]:

$$
\frac{\partial N_{i}}{\partial t}=-N_{i} \sum_{j} W_{i, j}+\sum_{j} W_{j, i} N_{j}+G_{i}(t),
$$

где $N_{i}$ - число электронов в $i$-й ячейке, $W_{i, j}-$ вероятность рассеяния из $i$-й ячейки в $j$-ю за счет $D A$ и $P A$ механизмов, $G_{i}$ - темп генерации электронов в $i$-й ячейке за счет внешнего воздействия.

При нахождения вероятностей $W_{i, j}$ надо иметь в виду, что из ячейки с большей энергией не все частицы могут рассеяться в состояние с меньшей энергией. Причина этого состоит в том, что частицы с большей энергией могут находиться в областях, где не может быть частиц с меньшей энергией, а рассеяние идет с сохранением координат электрона. Поэтому вероятность $W_{i, j}$ можно представить в виде

$$
W_{i, j}=W\left(E_{i}, E_{j}\right) g\left(E_{j}\right) f_{3}\left(E_{i}, E_{j}\right),
$$

где $f_{3}\left(E_{i}, E_{j}\right)$ - доля фазового пространства $i$-й ячейки, из которой электроны могут рассеяться в $j$-ю ячейку. Нетрудно получить аналитическое выражение для $f_{2}\left(E_{i}, E_{j}\right)$. Из-за его громоздкости мы его не будем приводить.

Для контроля точности численного решения были проведены расчеты с удвоенным числом ячеек (2300). Изменение результатов расчета функции распределения за счет удвоения числа ячеек имело величину менее процента.

\section{3. Результаты и обсуждение}

На рис. 1 представлены вычисленные зависимости от времени концентрации фотоэлектронов в делокализованных состояниях зоны проводимости GaAs c $N_{D}=3 \cdot 10^{15} \mathrm{~cm}^{-3}$ при различных уровнях компенсации и температуре $4.2 \mathrm{~K}$. Фотоэлектроны рождались с энергией 0.1 мэВ в течение 0.1 нс. Из рисунка видно, что зависимость от времени концентрации электронов близка к экспоненциальной. Поэтому время захвата электрона на заряженный донор определялось по наклону зависимости логарифма концентрации от времени.

На рис. 2 представлены вычисленные зависимости частоты каскадного захвата (величины, обратной времени каскадного захвата) от концентрации заряженных доноров в $\mathrm{GaAs}$ при $T=4.2 \mathrm{~K}$ для двух концентраций доноров $N_{D}=3 \cdot 10^{15}$ и $3 \cdot 10^{14} \mathrm{~cm}^{-3}$ для импульсного возбуждения. Кроме того, там представлены зависимость частоты каскадного захвата на кулоновские центры при импульсном возбуждении, найденная в работе (штриховая линия) [4], и зависимость, вычисленная по 
теории Абакумова-Переля-Яссиевич [3] для стационарного возбуждения для $N_{D}=3 \cdot 10^{15} \mathrm{~cm}^{-3}$ при захвате на диполи. В теории Абакумова-Переля-Яссиевич [3] частота каскадного захвата равна:

$$
\begin{aligned}
\frac{1}{\tau}= & N_{D}^{+} k_{\mathrm{B}} T\left[\int_{E_{i}}^{0} d E^{\prime} \frac{N_{D}^{+} \exp \left(E^{\prime} / k_{\mathrm{B}} T\right)}{B\left(E^{\prime}\right)}\right]^{-1} \\
& \times\left[\int_{0}^{\infty} d E g^{\prime}(E) \exp \left(-E / k_{\mathrm{B}} T\right)\right]^{-1},
\end{aligned}
$$

где $B\left(E^{\prime}\right)$ - коэффициент динамического трения (см. в работе [3] Приложсение 7), $g^{\prime}(E)$ - плотность состояний свободного электрона. В работе [3] было найдено выражение для $B(E)$ в случае каскадного захвата на диполи при рассеянии электронов на деформационном потенциале акустических фононов:

$$
B(E)=\left(\frac{e^{2}}{\kappa}\right)^{2} \frac{4 d m^{4}}{\pi^{2} \hbar^{7}} \frac{\Xi^{2}}{\rho}=\frac{8 d m^{4}}{\pi \hbar^{6}} \frac{s^{2}}{\mathbf{p}_{0}^{2} \tau_{D A}}\left(\frac{e^{2}}{\kappa}\right)^{2},
$$

где $\rho-$ плотность кристалла, $\Xi-$ константа деформационного потенциала, $s$ - скорость продольного звука, $\tau_{D A}$ - номинальное время рассеяния на деформационном потенциале акустических фононов [6], $\mathbf{p}_{0}-$ волновой вектор электрона с энергией продольного оптического фонона. Однако при выводе этого выражения авторы [3] при интегрировании (8) приняли, что $B(E)=B(0)$. Более точное выражение имеет вид

$$
B(E)=\frac{m^{4} s^{2} d}{\pi \hbar^{6} \tau_{D A} \mathbf{p}_{0}^{3}}\left(\frac{4 e^{2}}{\kappa}\right)^{2} I_{1}(E),
$$

где

$$
\begin{gathered}
I_{1}(E)=\int_{v_{1}}^{1} d v \frac{v}{2} \ln \left(\frac{\left(\mu_{1}-v\right)(1+v)}{\left(\mu_{1}+v\right)(1-v)}\right)-\left(\frac{v|E| \kappa d}{2 e^{2}}\right. \\
\left.+\left[\frac{|E| \kappa d}{4 e^{2}}\right]^{2} v^{2}\right)\left(\mu_{1}-1\right)+\left[\frac{|E| \kappa d}{4 e^{2}}\right]^{2} \frac{\left(\mu_{1}^{3}-1\right)}{3} \\
\mu_{1}=\sqrt{v^{2}+\frac{4 e^{2} v}{\kappa d|E|}}, \quad v_{1}=-\frac{2 e^{2}}{\kappa d|E|}+\sqrt{\left(\frac{2 e^{2}}{\kappa d|E|}\right)^{2}+1} .
\end{gathered}
$$

Важно отметить, что при выводе (10) в качестве потенциала $U(\mathbf{r})$ брался не потенциал диполя, а потенциал двух разноименных зарядов, располагающихся на расстоянии $d$ друг от друга.

Для рассеяния на пьезоэлектрическом потенциале акустических фононов вычисления приводят к следующему выражению для $B(E)$ :

$$
B(E)=\frac{8}{15} \frac{s^{2} m^{3}}{p_{0} \tau_{P A} \pi \hbar^{4}} \frac{\left(e^{2} d / \kappa\right)^{3 / 2}}{\sqrt{|E|}},
$$

где $\tau_{P A}-$ номинальное время рассеяния на пьезоэлектрическом потенциале акустических фононов [6].

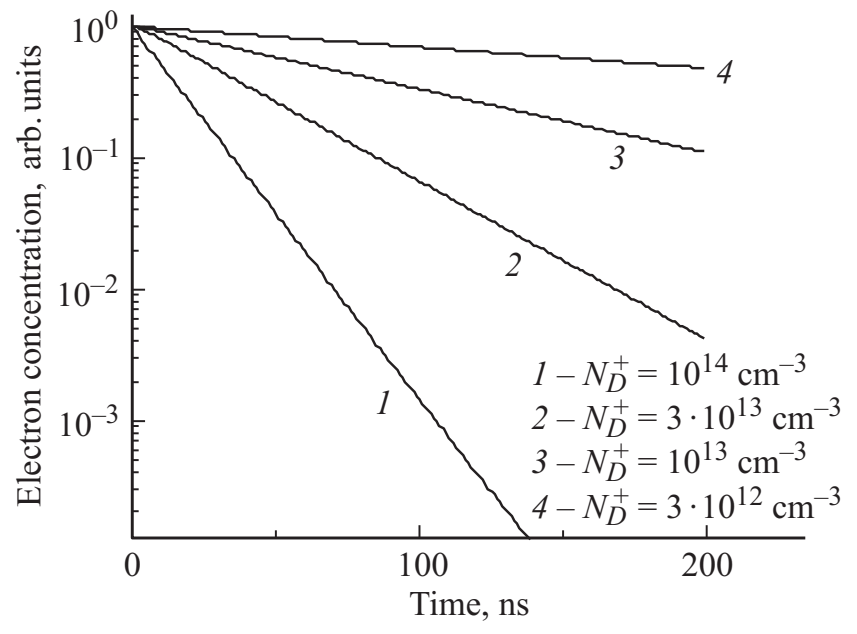

Рис. 1. Зависимость концентрации фотоэлектронов в делокализованных состояниях от времени при импульсном возбуждении в GaAs c $N_{D}=3 \cdot 10^{15} \mathrm{~cm}^{-3}$ при $4.2 \mathrm{~K}$.

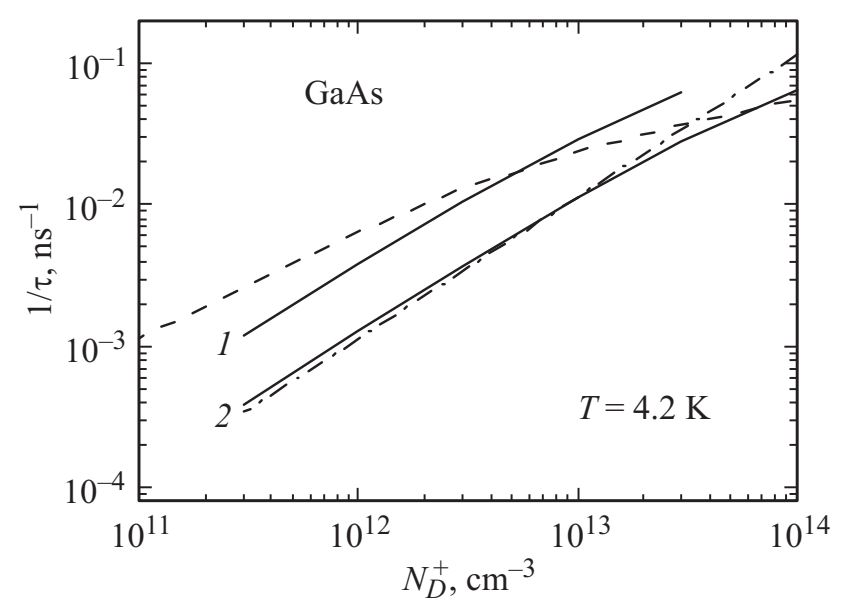

Рис. 2. Зависимости обратного времени захвата от $N_{D}^{+}$в GaAs. Сплошные линии 1 и 2 соответствуют $N_{D}=3 \cdot 10^{14} \mathrm{~cm}^{-3}$ и $N_{D}=3 \cdot 10^{15} \mathrm{~cm}^{-3}$. Штриховая линия соответствует захвату на кулоновские центры (эта зависимость вычислена методом, использованным в [4]). Штрихпунктирная линия получена с помощью теории Абакумова-Переля-Яссиевич для захвата на диполи при стационарном возбуждении при $N_{D}=3 \cdot 10^{15} \mathrm{~cm}^{-3}$.

Поскольку в GaAs электрон рассеивается на деформационном и пьезоэлектрическом потенциалах акустических фононов, то при расчете частоты каскадного захвата с помощью (8) для $B(E)$ была взята сумма $(10)$ и (11). Отметим, что замена плотности состояний $g^{\prime}(E)$ на плотность состояний (3) практически не изменяет результата (8).

Из рис. 2 видно, что частота каскадного захвата на диполи увеличивается при увеличении степени компенсации. Причина этого в увеличении дипольного момента пары заряженный донор-акцептор. Кроме того, видно, что зависимость частоты каскадного 


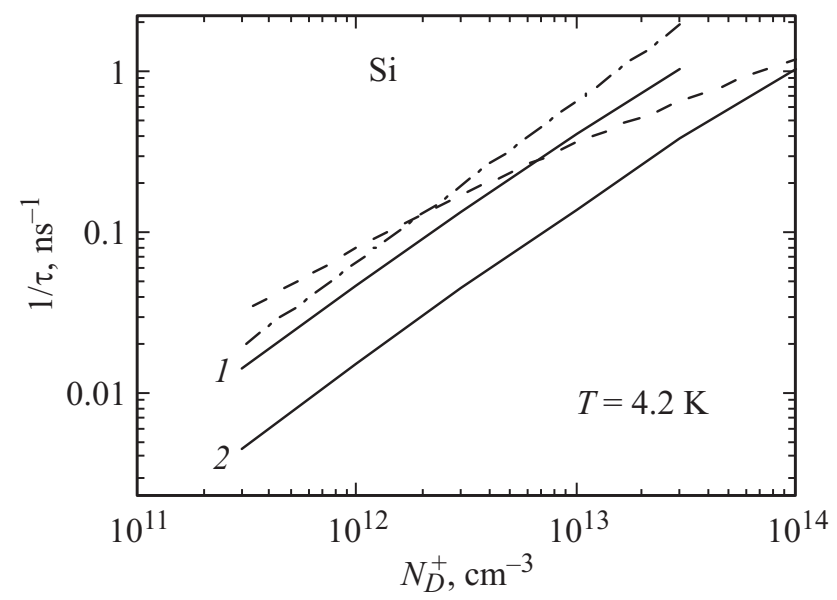

Рис. 3. Зависимости обратного времени захвата от $N_{D}^{+}$в $\mathrm{Si}$. Сплошные линии 1 и 2 соответствуют $N_{D}=3 \cdot 10^{14}$ и $3 \cdot 10^{15} \mathrm{~cm}^{-3}$. Штриховая линия соответствует захвату на кулоновские центры. Штрихпунктирная линия получена с помощью теории Абакумова-Переля-Яссиевич для захвата на диполи при стационарном возбуждении при $N_{D}=3 \cdot 10^{14} \mathrm{~cm}^{-3}$.

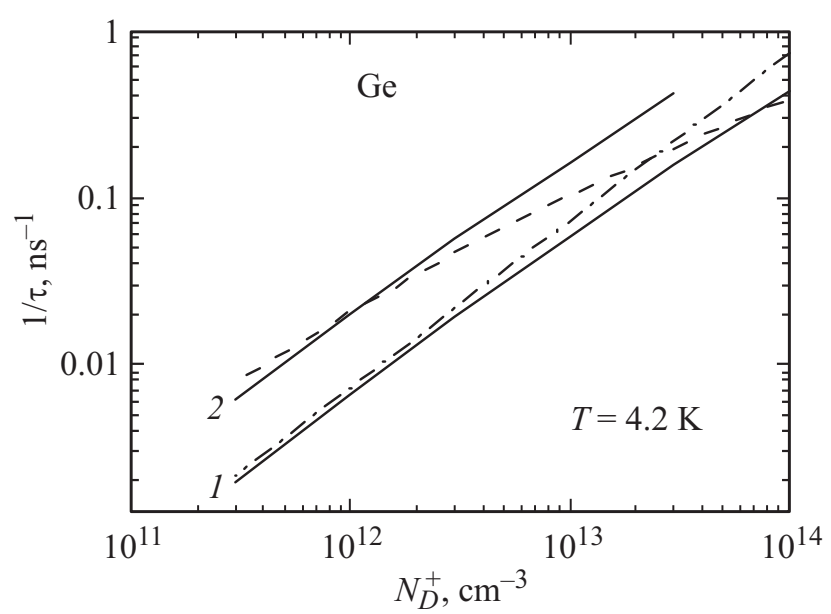

Рис. 4. Зависимости обратного времени захвата от $N_{D}^{+}$в Ge. Сплошные линии 1 и 2 соответствуют $N_{D}=3 \cdot 10^{14}$ и $3 \cdot 10^{15} \mathrm{~cm}^{-3}$. Штриховая линия соответствует захвату на кулоновские центры. Штрихпунктирная линия получена с помощью теории Абакумова-Переля-Яссиевич для захвата на диполи при стационарном возбуждении при $N_{D}=3 \cdot 10^{14} \mathrm{~cm}^{-3}$.

захвата на диполи от $N_{D}^{+}$близка к линейной при $N_{D}^{+} \ll 10^{13} \mathrm{~cm}^{-3}$. При $N_{D}^{+}>10^{13} \mathrm{~cm}^{-3}$ зависимость частоты каскадного захвата от $N_{D}^{+}$становится сублинейной. Отметим, что для захвата на кулоновские центры отклонение зависимости $1 / \tau\left(N_{D}^{+}\right)$от линейного закона начинается при меньшей концентрации: $N_{D}^{+} \sim 10^{12} \mathrm{~cm}^{-3}$. Из рис. 3 видно также, что при концентрациях $N_{D}^{+}>6 \cdot 10^{13} \mathrm{~cm}^{-3}$ для $N_{D}=3 \cdot 10^{15} \mathrm{~cm}^{-3}$ и $N_{D}^{+}>6 \cdot 10^{12} \mathrm{~cm}^{-3}$ для $N_{D}=3 \cdot 10^{14} \mathrm{~cm}^{-3}$ частота каскадного захвата на диполи при импульсном возбуждении больше таковой для захвата на кулоновские центры. Отметим, что в теории Абакумова-Переля-Яссиевич частота каскадного захвата на кулоновский центр в GaAs всегда выше, чем на диполь. В чем состоит причина увеличивающегося расхождения между временами захвата, вычисленными при импульсном и стационарном возбуждениях (между линией 2 и штрихпунктирной линией) с ростом $N_{D}^{+}$? Как показывает расчет, в случае импульсного возбуждения функция распределения в области делокализованных состояний вблизи нулевой энергии (откуда идет захват) в каждый момент времени зависит от энергии более плавно, нежели $\exp \left(-E / k_{\mathrm{B}} T\right)$. При выводе (8) функция распределения электронов предполагалась максвелловской [3]. Решая уравнение Фоккера-Планка, можно показать, что при стационарном возбуждении функция распределения является максвелловской для электронов с энергией, которая больше энергии фотоэлектрона при рождении [4]. Кроме того, следует отметить, что учет изменения плотности состояний электрона вблизи порога протекания, обусловленной присутствием диполей, слабо влияет на частоту каскадного захвата, найденную из (8). В случае каскадного захвата на кулоновские центры такое изменение плотности состояний существенно изменяло частоту каскадного захвата при концентрациях заряженной примеси $>10^{10} \mathrm{~cm}^{-3}[4]$.

На рис. 3 и 4 приведены вычисленные зависимости частоты каскадного захвата на диполи в кремнии и в германии при импульсном возбуждении и при стационарном возбуждении (согласно (8)-(10)). Там же приведены зависимости частоты каскадного захвата на кулоновские центры. Из рисунков видно, что в кремнии частота каскадного захвата примерно вдвое выше, чем в германии, и более чем на порядок выше, чем в GaAs, при тех же концентрациях диполей и доноров. Причина этого состоит в том, что времена релаксации энергии на акустических фононах электронов, участвующих в каскадном захвате, в $\mathrm{Si}$ выше, чем в $\mathrm{Ge}$, и в обоих этих полупроводниках выше, чем в GaAs.

Сопоставляя результаты работы [4] и данной работы, можно сделать вывод, что разница частот каскадного захвата на диполь для импульсного и стационарного возбуждений существенно меньше, чем такая же разница в случае захвата на кулоновский центр.

\section{4. Заключение}

В заключение приведем основные результаты работы. Вычислены зависимости частоты каскадного захвата на диполи от их концентрации в случае импульсного и стационарного возбуждений в $n$-GaAs, $n$-Si и $n$-Ge. Показано, что при достаточно больших концентрациях диполей частоты каскадного захвата для импульсного и стационарного возбуждений различны. Однако это различие не столь велико, как в случае захвата электронов на кулоновские центры.

Работа выполнена при финансовой поддержке РФФИ (проект № 16-02-00325). 
Функция $f_{2}(E)$ имеет вид

$$
f_{2}(E)= \begin{cases}\sqrt{|E|}, & E<-E_{0} \\ 2 \frac{E_{0}^{3 / 2}}{|E|^{3 / 2}\left(5-3 \frac{|E|}{E_{0}}\right)}\left\{1-\left(1-\frac{|E|}{E_{0}}\right)^{5 / 2}\right\}, & -E_{0}<E<0 \\ 2 \frac{E_{0}^{3 / 2}}{E^{3 / 2}\left(5+3 \frac{E}{E_{0}}\right)}\left\{\left(\frac{E}{E_{0}}+1\right)^{5 / 2}-1\right\}, & 0<E<-E_{0} \\ \frac{E_{0}^{3 / 2}}{E^{3 / 2}\left(5-\left(\frac{E_{0}}{E}\right)^{3 / 2}\right)}\left\{\left(\frac{E}{E_{0}}+1\right)^{5 / 2}-\left(\frac{E}{E_{0}}-1\right)^{5 / 2}-1\right\}, & E_{0}<E .\end{cases}
$$

\section{Список литературы}

[1] A.L. Efros, B.I. Shklovsky, I.J. Janchev. Phys. Status Solidi B, 50, 45 (1972).

[2] В.Н. Абакумов, В.И. Перель, И.Н. Яссиевич. ЖЭТФ, 72, 674 (1977).

[3] V.N. Abakumov, V.I. Perel, I.N. Yassievich. Nonradiative Recombination in Semiconductors (Elsevier Science Publishers, Amsterdam, 1991).

[4] В.Я. Алешкин, Л.В. Гавриленко. ЖЭТФ, 150, 328 (2016).

[5] Л.П. Питаевский. ЖЭТФ, 42, 1326 (1962).

[6] В.Ф. Гантмахер, И.Б. Левинсон. Рассеяние носителей тока в металлах и полупроводниках (М., Наука, 1984).

Редактор Г.А. Оганесян

\section{Cascade capture of electrons by charged dipoles in weakly compensated semiconductors}

V.Ya. Aleshkin, L.V. Gavrilenko

Institute for Physics of Microstuctures of the Russian Academy of Sciences, 603950 Nizhny Novgorod, Russia

Abstract The times of cascade capture of an electron by a charged donor-acceptor dipole are calculated for the case of pulsed and stationary excitations of impurity photoconductivity in GaAs, $\mathrm{Ge}$, and $\mathrm{Si}$. It is shown that for a charged impurity concentration greater than $10^{13} \mathrm{~cm}^{-3}$, the dependence of the cascade-capture frequency on the concentration of a charged impurity becomes sublinear in the semiconductors under consideration. 